\title{
Meditations among the Tombs: In a Letter to a Lady de James Hervey, traducida al francés en 1770 por Mme d'Arconville
}

\section{(James Hervey's Meditations among the Tombs: In a Letter to a Lady, translated into French in 1770 by Mme d'Arconville)}

BEATRIZ MARTÍNEZ OJEDA

Fecha de recepción: 29 de agosto de 2013

Fecha de aceptación: 30 de septiembre de 2013

Resumen: La denominada "Graveyard School" o Poesía de las tumbas surge en pleno declive de la estética literaria augusta que florece a comienzos del siglo XVIII. Este giro implica el abandono paulatino de los paisajes idílicos de la Arcadia como consecuencia de la óptica humanitaria que define la llamada Cultura de la sensibilidad de mediados de siglo.

La idea subyacente de esta modalidad poética sombría y melancólica se concibe como una reacción al progreso social que aliena a las clases sociales y margina a las clases bajas y campesinas a la más absoluta pobreza. Los poetas de la Poesía de las tumbas tienen por objeto denunciar la precariedad y demandar conmiseración a aquellos que gozan de riquezas. La muerte, la tumba y el cementerio se tornan en imágenes recurrentes que hacen alusión a la mutabilidad de la materia y la fugacidad de la existencia. Con estas, los poetas del cementerio pretenden conmover a los ambiciosos y los alientan a que contemplen la salvación espiritual de los miserables así como la suya.

El hecho de traducir las obras más aclamadas de los poetas de la Poesía de las tumbas, en nuestro caso, Meditations among the Tombs: In a letter to a Lady (1746) del prosista y clérigo James Hervey, supone estrechar lazos entre la modalidad lúgubre inglesa, única en su estética, y la de los países circundantes, en concreto Francia. La versión francesa de Mme d'Arconville (1770) es un ejemplo ilustrativo de este intercambio intertextual e intercultural. Su traducción destaca por la sublime belleza de sus versos y la fidelidad que muestra con respecto al texto original. Sin embargo, su talento siempre ha estado bajo la sombra de otros traductores contemporáneos de gran reconocimiento. 
El objetivo principal es analizar las características principales que moldean la versión francesa de Meditations among the Tombs: In a letter to a Lady.

Palabras clave: "Graveyad School" o Poesía de las tumbas, Melancolía, Muerte, Traducción.

Abstract: The so-called Graveyard School emerged in the twilight of the Augustan literary aesthetics, which flourished during the early 1700's. This turn meant a change from the the idyllic landscapes of Arcadian poetry owing to the humanitarian look that characterised the Culture of Sensibility in the mid-century England.

The underlying leitmotiv of this gloomy and melancholy poetic mode was the reaction to the idea of material progress, which estranged the social echelons making the poor class even poorer and devoid of rights. In light of that, these poets devoted themselves to denouncing precariousness and to urging the upper classes to commiserate with the poor. Death, the tomb and the graveyard were recurring images representative of the mutability and fugacity of worldly life, which meant to move the reader deeply and instigate him/her to meditate upon the Christian salvation of the silenced other and upon his/her own.

Translating these poets' masterpieces, particularly Meditations among the Tombs: In a letter to a Lady (1746) by James Hervey, bridged the gap between the unique English melancholy aesthetics and that of the neighbouring countries, especially France. Mme d'Arconville's version (1770) is an example of this intertextual-intercultural exchange and it outstands for its sublimity and fidelity to the original text. However, her talent has been eclipsed by other greatly acclaimed translators of the period.

The primary objective of this paper is to analyse the main features that give shape to Mme d'Arconville's French translation of the original version of Meditations among the Tombs: In a letter to a Lady.

Key words: Graveyad School, Melancholy, Death, Translation.
INTRODUCCIÓN
El prerromanticismo en Francia se originó en gran medida por la influencia de la producción literaria extranjera, en especial, por el influjo británico, así como por las traducciones al francés de textos ingleses que con gran éxito se difundían al responder al cambiante gusto del público francés. La corriente racionalista, que había imperado en el país vecino durante los siglos XVII y primer mitad del siglo XVIII, dejaba progresivamente paso a una nueva estética sentimentalista que, en 
contraposición con aquélla, entendía la expresión del sentimiento individualista como el modo idóneo de crear literatura.

La literatura inglesa se erigía en este sentido como el centro neurálgico europeo por excelencia. En concreto, a partir de 1740, el género lírico se impregnaba de una estética melancólica que instigaba a la reflexión de la condición humana y las actividades del hombre, y que armonizaba el didactismo religioso heredado del puritanismo con el deleite de la poesía. La crítica francesa, hastiada de la tradición racionalista y ansiosa por la renovación de las arraigadas tendencias literarias, encuentra en las creaciones fúnebres de los poetas de Graveyard School o L'École des Cimitières, especialmente en Thomas Parnell, Edward Young, James Hervey y Thomas Gray, su fuente de inspiración.

Los temas recurrentes que teñían de un aire sepulcral esta modalidad poética son la enfermedad física y social, la muerte, la tumba y la oscuridad de la noche, elementos de gran realismo que inspiran a estos poetas a plasmar en sus melancólicos versos sus más profundas críticas, advertencias y contemplaciones:

Comme, d'autre part, l'obscurité de la nuit est éminemment favorable dans leurs ouvrages avec l'élément sépulcral. Un prête de champagne méditant la nuit parmi des tombeaux; ainsi peut se résumer assez exactement l'école anglaise des cimetières (Van Tieghem, 1921: 11).

Las traducciones, en particular las francesas, de las obras inglesas de tonalidad sepulcral se convirtieron en un canal de transmisión e influjo de esta estética que otorgó prominencia a los poetas de la "Poesía de las tumbas". Parnell, Young, Hervey y Gray se establecieron como los precursores modelo del timbre gótico del romanticismo europeo de la primera mitad del siglo XIX.

Aimé Feutry (1720-1784) da buena cuenta de la "invasión" literaria inglesa; en 1751 el poeta, imitando a James Thompson, Edward Young y, en especial, a Alexander Pope, concibe la que sería su traducción más sobresaliente: Épître d'Héloïse à Abelard, obra que poco después CharlesPierre Colardeau retomaría con la esperanza de revitalizar el género de la heroida.

Le Tourneur (1736-1788) ha sido considerado tradicionalmente por la crítica como el intérprete por excelencia de la producción literaria de la "Poesía de las tumbas"; dicho autor y traductor francés, que conocía la repercusión inglesa en la literatura de su país, determina publicar en 1769 una traducción en prosa -aunque sería más riguroso calificarla de imitación- de la obra de Edward Young, The Complaint; or Night Thoughts on Life, Death and Immortality (1742), versión al francés que obtuvo un 
rotundo éxito en Francia, que llegó a sobrepasar incluso al de la obra original en Inglaterra.

Haciéndose eco de estas nuevas tendencias literarias y tratando de granjearse la misma suerte que Le Tourneur, no pocos autores franceses se embarcaron en la empresa de dar difusión por medio de la traducción a esta incipiente estética que preludiaba la corriente romántica. Este es el caso de Mme d'Arconville, que publica en 1770 -poco después vería la luz la traducción de Le Tourneur para la misma obra, que lleva por título Méditations d'Hervey-, la versión al francés de Meditations among the Tombs: In a Letter to a Lady (1746) de James Hervey, único prosista de la "Poesía de las tumbas". Desafortunadamente, la reputación que precedía a Le Tourneur hacía de la versión de la autora una obra sin mérito ni interés para el público francés, por lo que, Méditations sur les tombeaux de Mme d' Arconville, pese a su valor literario y traductológico incuestionable, ha sido relegada al olvido sin otra utilidad que la de corroborar la influencia inglesa en la literatura francesa de finales del siglo XVIII.

\section{LA "POESÍA DE LAS TUMBAS": DIDACTISMO Y SENTIMIENTO DEVOCIONAL ${ }^{1}$}

El marco religioso del siglo XVII se configura a favor del dogma protestante, en el que se sustituye la institucionalización y formalización de los preceptos calvinistas por una lectura privada y guiada de los textos sagrados en la que los sermones, sobre todo los fúnebres, adquieren un papel fundamental, puesto que predican sobre la brevedad de la vida, la banalidad del mundo material y la preparación obligatoria del individuo para afrontar el óbito y ser obsequiados con la salvación del alma. ${ }^{2}$

Como consecuencia, los manuales devocionales impresos de carácter didáctico debilitan la tradición oral de la Iglesia, los cuales

\footnotetext{
${ }^{1}$ El clérigo y prosista inglés James Hervey, (1714-1758), en su epístola Meditations among the Tombs (1746), esgrime el cementerio de una iglesia abandonada en el pueblo de Kilkhampton como principal escenario. Para Hervey, el cementerio es el lugar más sagrado, puesto que deja huella en el visitante y lo incita a reflexionar sobre la banalidad de la vida terrenal, la muerte, la condena y la salvación del espíritu, temas que rezuman el sentimiento puritano del siglo anterior en una corriente de pensamiento cuyos pilares son el racionalismo, el empirismo y el escepticismo. En A Winter Piece, obra en prosa compilada en Meditations and Contemplations (1746-1747), Hervey comunica el cariz religioso de la fe, el cual cultiva como ferviente discípulo de la poesía devocional desarrollada por John Dennis. El prosista comienza con una sucinta descripción de las estaciones, lo que recuerda la trascendencia de The Seasons (1730) del poeta escocés James Thomson, contemporáneo de Hervey y representante exponencial de la denominada "Poesía de la Naturaleza" de principios del siglo XVIII. A Winter Piece acopia las connotaciones clásicas del ciclo de la vida empleadas por el escocés, sin embargo, Hervey las tiñe de una gradación meditativo-religiosa que se cimienta en la fe y el sentimiento, propio de la "Poesía religiosa".

${ }^{2}$ Los sermones fúnebres datan del siglo XIII, los cuales ya anunciaban como tema principal la oración para la salvación del alma y la reflexión sobre la muerte, el Juicio Final, el cielo y el infierno.
} 
aconsejan un constante estudio de la palabra divina para alcanzar fructíferos resultados concernientes a la virtud y la moral espiritual. Impregnándose del espíritu consagrado en la transmisión de la genuina fe mediante la página impresa, la poesía de índole devocional aúna el mensaje del sermón de los textos bíblicos, la instrucción íntima del ánima y el deleite de transportar al lector a los Altos Dominios no sólo de la imaginación, sino también del sentimiento individual, de la subjetividad y de los principios de la fe:

From the early to mid-eighteenth century, we can discern a movement away from an exclusively public and active form of religious worship to a cloistered private devotion centered upon the individual reading, one that acknowledged the valuable possibilities of poetic edification. It is difficult to envisage the flourishing taste for graveyard poetry during this period without this development (Parisot, 2011, 89).

Esta concepción armoniosa entre el género poético y la religión coincide con la formulación estético-religiosa que propone John Dennis en su The Grounds of Criticism in Poetry (1704), en la que da importancia a las pasiones que tanto la creencia como la poesía engendran como el canal trascendental para la monitorización del individuo. Por consiguiente, el doble fin del arte, esto es instruir y deleitar, heredado de la tradición clásica, se reduce con Dennis a la siguiente teorización, es decir, el placer que emana de la pasión es crucial para la reconstitución y la iluminación del espíritu:

The greater and more comprehensive the Soul is, which reflects upon the Idea of God, the more that Idea must fill that Soul with Admiration: it follows. That as great Passion only is the adequate Language of the greater Poetry, so the greater Poetry is only the adequate Language of Religion (Dennis, 1718: 426-427).

El auge del sermón fúnebre en texto impreso hasta bien entrados el siglo XVIII poco a poco ve su ocaso, "from the late seventeenth-century through to the mid-eighteenth century" (Parisot, 2011: 91), mas debido a su preciado valor instructivo y reconfortante, además de la creciente mortandad, factor que intensifica el recelo a la muerte y la preocupación por la salvación individual, el escepticismo que se sucede del racionalismo y la experimentación analítica pura, el cambio social y económico de la industrialización, la esencia de estos valiosos predicamentos se materializa, en primer instancia, con la restauración de la elegía fúnebre y después con la estética lúgubre de la referida "Poesía de las tumbas", que recoge las 
experiencias individuales de la pérdida de los seres queridos en una burbuja imaginativa en la que vibra la melancolía y el didactismo religioso. ${ }^{3}$

En la tesitura de la primera mitad del siglo XVIII, la estética de la "Poesía de las tumbas" esgrime la temática de la transitoriedad de la fama y la vida, el deleite que se deriva de la soledad y del aislamiento, y la inexpugnable muerte. A ésta se adiciona el lienzo de un mundo de castillos derruidos por el tiempo, catedrales góticas desmoronadas, abadías e iglesias en ruinas, sombríos cementerios y osarios a la luz de la medianoche que dejan vislumbrar los estragos de la descomposición de la materia y la aridez de la adusta muerte, elementos e ideas que constituirán las bases de la novela gótica de las últimas décadas de este siglo. De manera similar, perpetúa la aleación entre las descripciones paisajísticas y el subjetivismo, así como también el didactismo religioso y las reflexiones críticas a la esfera social. Es ilustrativo el siguiente fragmento tomado de Meditations among the Tombs: In a Letter to a Lady (1746) de James Hervey, obra en prosa poética que nos concierne: ${ }^{4}$

To these how calm was the Evening of Life! In what a smiling Serenity did their Sun go down! When their Flesh and their Heart failed, how reviving was the Remembrance of an All-sufficient Redeemer; once dying for their Sins, now risen again for their Justification! How chearing the well-grounded Hope of Pardon for their Transgressions; and Peace with GOD, through JESUS CHRIST our LORD! How did this assuage the Agonies, and sweeten the Bitterness of Death! - Where now is Wealth, with all her golden Mountains? Where is Honour, with her proud Trophies of Renown? Where are all the vain Pomps of a

\footnotetext{
${ }^{3}$ Críticos como Ralph Houlbrooke postulan que las normas del decoro que dan forma a la sociedad inglesa de la llustración evitan la democratización de los desafortunados sucesos de la esfera privada, "Eighteenth-century polite society put in the prívate sphere the sorts of evidence which had been laid out in the best funeral sermons of an earlier generation" (Houlbrooke, 1998: 326).

${ }^{4}$ Refleja la temática que propone Parnell ya a comienzos del siglo XVIII; ésta actúa como vertiente opuesta al patrón poético del Neoclasicismo y va a convivir con éste hasta su desembocadura en el Prerromanticismo inglés de finales de siglo. Hervey, en prosa poética, plasma los elementos lúgubres con el aspecto religioso del cristianismo: el ciclo vida-muerte como viaje, la luz-oscuridad, la noche y el día, la melancolía, el sufrimiento, la inevitabilidad de la muerte, los estragos del tiempo, el silencio, locuaz en sus enseñanzas, el pecado y la salvación del hombre mediante el carácter privado de la confesión y comunión con Dios. Igualmente, la obra se nutre del sentimiento sublime del horror provocado mediante la figura de Dios, "Hervey explains that timid people are frightened of imaginary Horrors of the Night when they should be afraid of God" (Spacks, 1962: 25). El locus eremus de las ruinas y las tumbas, la iglesia abandonada, la figura del viajero errante y la soledad, sirve como remembranza de tempus fugit y del memento mori. La naturaleza muerta y silente de la iglesia, reflejo de la decadencia de la naturaleza humana y de las tumbas, es compañera y fiel instructora para e errante.
} 
deluded World? Can they administer any Support in this last Extremity? Can they compose the affrighted Thoughts, or buoy up the departing Soul amidst all the Pangs of Dissolution? The Followers of the Lamb seem pleased and triumphant even at their last Gasp, "GoD's everlasting Arms are underneath" their fainting Heads. His Spirit whispers Peace and Consolation to their Consciences (Hervey, 1746: 37).

La "Poesía de las tumbas" hace referencia principalmente al conglomerado de poemas de timbre elegíaco, una poesía "plus intime et plus sérieuse [...] d'un aspect funèbre et mélancolique" (Van Tieghem, 1921: 7) de tono moral, que gira en torno a la meditación metafísica sobre la transitoriedad de la existencia, la ineludible muerte y el consuelo que la fe cristiana promete después de la muerte, los cuales datan de la primera mitad del siglo XVIII. ${ }^{5}$ Sin embargo, la compilación de las obras de James Hervey, clérigo inglés, es una excepción, puesto que éste recupera el género de la prosa para tratar el timbre y la temática oscura de esta estética:

[It] incorporates a veritable host of popular poetry and prose of the early to mid-eighteenth century (...) the very popularity of this lachrymose taste is perhaps the school's most distinctive feature (Parisot, 2011: 85) ${ }^{6}$.

La génesis de la modalidad inglesa se traduce como una transformación del subgénero de la elegía fúnebre del siglo XVII, siendo ésta "an ideal of personal expression of grief [which] begins to replace critical self-restraint" (Pigman, 1985: 3$).^{7}$ Por consiguiente, este subgénero

\footnotetext{
${ }^{5}$ Como expone Van Tieghem, la mayoría de estos poetas profesan en el ámbito divino de la religión y tienen como fin convertir e instruir al escéptico, utilizando experiencias palpables y comunes a la humanidad como la enfermedad, la muerte y la tumba, "tous ces poètes sont des ministres de la religion (...) Ils appliquent leur talent d'écrire á des sujets que leur suggèrent les devoirs de leur charge et l'expérience de leur ministère. Ils veulent être utiles, réformer le pécheur, convertir l'incrédule; leurs arguments sont tirés des spectacles qu'ils ont constamment sous les yeux: la maladie, la mort, le tombeau" (Van Tieghem, 1921: 11).

${ }^{6}$ Desde finales del siglo XVII en Alemania, el poeta lírico Christian Hoffmann von Hoffmannswaldau (1616-1679) "representa en 1697, veinte años después de la muerte de su autor, un paseo por un cementerio con la evocación de héroes antiguos, Alejandro y César. Entre sus contemporáneos, el lírico y dramaturgo Andreas Greif (1616-1664), con una lírica sombría y melancólica escribirá Kirchhofsgedanken (Meditaciones sobre el cementerio y la última morada de los difuntos, 1656), en el que expresa su sentimiento trágico hacia la fragilidad de la existencia y hacia la vanidad del mundo (García Peinado y Vella, 2007: 35).

${ }^{7}$ López-Folgado remarca que "las causas iniciales de este movimiento no están tan claras [...] lo que sí es cierto es que la denominada poesía que aborda la meditación nocturna alrededor de los cementerios y tumbas es original del siglo dieciocho inglés, a pesar de que haya claros 
sombrío se establece como puente entre el ethos de la elegía del siglo anterior y el adviento de la melancolía del Romanticismo, "[it] bridged the transition between the elegy proper [...] and the melancholy of nascent Romanticism" (Draper, 1967: 4). Igualmente, esta se considera una manifestación de la reinterpretación del Protestantismo desde una óptica puritana.

La estética del cementerio se parcela en dos períodos: la etapa de los antecedentes y la de los poetas modelos, Robert Blair, James Hervey y Thomas Gray. ${ }^{8}$ Entre los precursores destacan John Sheffield (1648-1721) y The Temple of Death (1695), John Cutts (1661-1707) y On the Death of the Queen (1695), John Hopkins (1675-?) y The Victory of Death (1698) o Thomas Parnell (1679-1718) en su A Night Piece on Death (1715), extenso poema compuesto por noventa octosílabos rimados. ${ }^{9}$ A este último se le reconoce como precursor de la "Poesía de las tumbas", puesto que firmemente responde a la estética desarrollada por esta escuela.

\section{Meditations SuR LES tombeauX De Mme d'ARConViLle}

La traducción al francés de Meditations among the Tombs: In a Letter to a Lady (1746) realizada por Mme Thiroux d'Arconville venía a suplir el vacío literario sobre James Hervey en Francia, del que sólo se había versionado parcialmente en 1757 su obra Contemplations on the Night (1747).

No pocos señalan erróneamente el año 1771 como fecha de publicación de la traducción de Méditations, e indican, asimismo, que esta vio la luz un año después de que Le Tourneur publicara su versión para la misma obra inglesa ${ }^{10}$; en este sentido, Van Tieghem trata de aclarar dicha

indicios de 'intertextos' precedentes en la llamada 'melancolía isabelina' presente en poetas como John Donne o Edmund Spencer" (López Folgado, "Los cementerios de aldea, un paraje compartido en la literatura europea: de Thomas Gray a Leonor de Almeida y Unamuno", Coloquio de Literatura Comparada, 2012).

${ }^{8}$ No obstante, se han registrado otros posibles antecedentes tales como el poeta metafísico galés Henry Vaughan (1622-1695), "The Charnel-House", John Rawlet (1642-1686), Midnight Meditations (1687), Thomas Flatman (1637-1688), A Dooms-Day Thoughts (1659) y Isaac Watts (1674-1748), "Death and Eternity". (García Peinado y Vella, 2007: 2).

${ }^{9}$ Se dice que Parnell acostumbraba a dar paseos, acompañado por la soledad de la oscuridad de la noche, por el cementerio, y entre los moradores en sus tumbas, que le instigan a meditar sobre el carácter universal de la muerte, la resignación a la inevitable hora, la inmortalidad de alma y la futilidad de las ceremonias fúnebres, "La vue des tombeaux lui suggère des réflexions sur l'égalité des hommes devant la mort, la résignation nécessaire á l'heure suprême, l'immortalité de l'âme, la vanité des rites et des pompes funèbres" (Van Tieghem, 1921: 12).

10 A modo ejemplo, señalamos el tomo IV de La France Littéraire ou Dictionnaire Bibliographique de J. M. Quérard (p. 100), en el que el autor señalada equivocadamente que la traducción de Mme d'Arconville fue publicada un año más tarde que la de Le Tourneur. 
confusión sosteniendo que, aunque la versión de Arconville fue publicada en 1771, fue creada el 21 de mayo de 1770:

De celles-ci [Méditations], il a paru deux traductions qui portent la même date (1171); mais en réalité, un intervalle assez long les séparait pour que le second traducteur, Le Tourneur, ait pu connaître et critiquer le travail de son devancier. Celui-ci, ou plutôt celle-ci, Mme Thiroux d'Arconville, publiait cette année-là, sous le voile de l'anonyme, les Méditations sur les Tombeaux, par Hervey, en un volume dont le privilège est daté du 21 mars 1770 (Van Tieghem, 1921: 66).

No obstante, resulta un tanto desconcertante, teniendo en consideración las palabras de Van Tieghem, que la traducción de la autora francesa saliera en prensa en 1771, cuando ya se aludía a ella en la primera parte del tomo VIII del Journal Encyclopédique ou Universel con fecha de 15 de noviembre de 1770 (pp. 53-63) y en el tomo $V$ de L'Année Littéraire de 1770 (pp. 23-24).

De hecho, el mismo Le Tourneur hace referencia en su traducción a la versión de su predecesora, aprovechando la ocasión para justificar la determinación de abreviar la suya y para desmerecer aquello que le resulta desacertado de la de Mme d'Arconville:

On trouvera que j'ai beaucoup abrégé les Tombeaux, si on en juge sur l'original, ou même sur une traduction qui a précédé la mienne. Je suis loin de vouloir rabaisser son mérite; mais je ne l'ai point imitée dans les emprunts qu'elle a faits des Nuits, dans les morceaux qu'elle a ajoutés d'imagination; et dans les longueurs qu'elle a laissé subsister (1771: 48)

Al margen de esta evidente contradicción, hemos de indicar que Méditations sur les tombeaux de Mme Thiroux d'Arconville fue publicada en un único tomo de 160 páginas, que evidencia una distribución por capítulos a los que la autora otorga un título que trata de resumir el contenido de los mismos, contraviniendo así la fragmentación epistolar de la obra de Hervey. Arconville se pronuncia al respecto en los siguientes términos:

Il est à propos d'avertir le public que pour mettre plus d'ordre dans ces Méditations, on a cru devoir les diviser suivant les différents sujets qui en font la matière. Cette forme d'ailleurs, sert à soulager la mémoire du Lecteur, et lui procure la facilité de choisir la Méditation qui pourra I'affecter davantage. Cette division n'est point marquée dans l'original; mais du reste, on a suivi le plan de l'Auteur (Ibidem, XIV). 
Del mismo modo, Arconville se permite la licencia de añadir información de su propia minerva, argumentando en el prólogo de su versión que el único propósito que persigue es el de facilitar la comprensión por parte del público francés:

On s'est permis aussi d'étendre et de développer certains pensées, pour mieux faire sentir, d'agrandir certains tableaux, et d'y ajouter quelques traits que l'imagination même désire à la lecture de cet ouvrage (Arconville, 1770: XV).

Sin embargo, comprobamos que más allá de explicitar o, incluso, parafrasear ideas del texto original que podrían resultar un tanto confusas para el lector meta, la traducción francesa revela un sinfín de adiciones que, lejos de aclarar el contenido, únicamente ayudan a adornar y embellecer el texto meta. No obstante, a pesar de que la autora se rija por sus propios criterios estéticos, su traducción sigue siempre la pauta del original sin distanciarse significativamente del contenido del mismo. Por ende, podríamos afirmar que se trata de una traducción libre, en tanto que, pese a discurrir hacia un estilo más personal, respeta el sentido del texto original.

A modo de ejemplo de tales adiciones, trascribimos un fragmento que ilustra la recreación estilística personal de Arconville, mas que no vulnera la semántica de Meditations among the Tombs:

O! how amiable is Gratitude! Especially, when it has the supreme Benefactor for its Object. I have always looked upond Gratitude as the most exalted Principe that can actuate the Heart of man. It has something noble, disinterested, and (if I may be allowed the Term) generously devout (p. 3).

O que la reconnoissance est estimable, sur-tout quand elle a pour objet le Bienfaicteur suprême! C'est le sentiment le plus noble \& plus exalté qui puisse mouvoir le cœur de l'homme. Il a je ne fais quoi de grand, et (si l'on peut s'exprimer ainsi) d'une piété généreuse (p. 4).

El extracto francés evidencia un contenido simétrico al del texto original, pese a que la autora francesa no ha respetado la disposición sintáctica de las dos primeras oraciones del fragmento inglés (O! how amiable is Gratitude! Especially, when it has the supreme Benefactor for its Object), que ha fusionado en una sola oración exclamativa (O que la reconnoissance est estimable, sur-tout quand elle a pour objet le Bienfaicteur suprême!), y a pesar de haber convertido en una cláusula impersonal (C'est le sentiment le plus noble \& plus exalté qui puisse mouvoir le cœur de l'homme), la tercera frase inglesa cuyo sujeto es la primera 
persona del singular (I have always looked upond Gratitude as the most exalted Principe that can actuate the Heart of man).

Sin embargo, en ocasiones, el discurso de Thiroux d'Arconville se torna tan descriptivo y tan plagado de florituras que la confrontación entre original y texto meta se convierte en una ardua tarea:

On this Hand is lodged one, whose Sepulchral Stone tells a most pitiable Tale indeed! Well may the little Images, reclin'd over the sleeping Ashes, hang down their Heads with that pensive Air! None can consider so mournful a Story without feeling some Touches of sympathizing Concern (p. 21).

Sur un des bas côtés de l'Église, j'aperçus un sépulcre, dont l'Épitaphe annonçoit un événement tragique. Des figures en marbre sont appuyées sur les quatre coins de la tombe, dans l'attitude de I'affliction: elles ont l'air pensif et désolé; à leurs pieds sont éteintes les torches croisées de l'Amour et de l'Hymen; la douleur tient leurs têtes penchées, et leurs regards fixés à jamais sur cette pierre immobile ( $\mathrm{p}$. 23).

En este caso, comprobamos que Arconville no sólo ha incorporado información no contenida en el original, sino que también obvia parte de ella; así pues, la disimetría entre ambos fragmentos es tal que difícilmente la versión francesa pueda ser considerada como traducción del extracto inglés.

Por otro lado, la versión francesa revela igualmente el propósito explicativo de la autora, quien trata de explicitar aquella información que considera susceptible de confusión para el lector meta:

Among these confused Relicks of Humanity, there are, without doubt, Persons of contrary Interests, and contradicting Sentiments (p. 12).

Parmi ces cadavres confus, il se trouve sans doute des hommes qui, durant le court trajet de leur vie, étoient animés par des intérêts opposés et des sentiments contraires (p. 12)

El anterior binomio demuestra que la traductora simplifica la redacción del sintagma inglés "Relicks of Humanity", suprimiendo la metáfora empleada por James Hervey y empleando el término al que rigurosamente se refiere el autor inglés con dicha figura literaria, "cadavres".

Las omisiones no suelen ser frecuentes en la versión francesa, sin embargo, constatamos cierta tendencia por parte de la traductora a obviar las notas a pie de página del original, así como los poemas insertados en el texto, tanto de creación propia como ajena; como ejemplo de este último 
caso, mencionamos la omisión de los versos 202-204 extraídos del primer poema de The Seasons, "Winter" (1726), de James Thomson, que Hervey incorpora en su obra para secundar su declamación a las banalidades de la vida terrenal:

For now, ye lying Vanities of Life!

Ye ever-tempting, ever-cheating Train!

Where are ye now? And what is your Amount?

Otro de los rasgos que caracterizan la versión de Arconville es que en ninguna ocasión trasvasa la grafía mayúscula y cursiva que con frecuencia emplea James Hervey para dar relevancia a ciertos términos de Meditationsk por su efecto poético. La autora opta por dar homogeneidad ortotipográfica a su versión, tal y como evidencian los fragmentos que transcribimos a continuación:

Examining the Record of Mortality, I found the Memorial of a promiscuous Multitude. They were huddled together, without any Distinction of Rank or Seniority. None were ambitious of the uppermost Rooms, or chief Scats, in the House of Mourning (p. 11).

En examinant ces monuments déplorables, qui sembloient se disputer mes regards, je ne trouvai d'abord que le mémorial d'une multitude de corps confondus pêle-mêle sous ces tombes funèbres. Ils habitoient ensemble, sans avoir égard aux rangs et aux titres. Aucun n'étoit ambitieux d'avoir une place plus distinguée dans cette demeure ténébreuse (p. 11).

En último término, es preciso indicar que Mme d'Arconville no suele incurrir en graves alteraciones semánticas, puesto que, aunque no se ciña estrictamente a la sintaxis y al léxico del texto inglés, la traductora, como señalamos anteriormente, concibe su narración siguiendo las directrices semánticas de la obra original.

\section{CONCLUSIÓN}

La traducción al francés de Meditations among the Tombs realizada por Mme Thiroux d'Arconville en 1770, así como las numerosas versiones de obras inglesas que fueron publicadas desde la segunda mitad del siglo XVIII en Francia, dan buena cuenta de la notable influencia de la "Poesía de las tumbas" en la historia literaria del país vecino.

Pese a que la figura del único prosista de la "Graveyard School", James Hervey, fuera eclipsada por la trayectoria de algunos miembros adscritos a su mismo movimiento literario, -especialmente la de su 
coetáneo Edward Young con su The Complaint; or Night Thoughts on Life, Death and Immortality (1742) - , su obra fue acogida de buen grado entre el público francés, que ansiaba seguir deleitándose con la producción literaria fúnebre y sentimentalista que se cultivaba del otro lado del Canal de la Mancha.

Así pues, la versión de Mme d'Arconville, que por responder al gusto imperante de la época podía haberse granjeado un gran éxito, queda relegada al olvido al publicarse paralelamente a la traducción para la misma obra inglesa del intérprete por excelencia de la "Graveyard School", Pierre Le Tourneur.

A pesar de haber pasado casi inadvertida ante los ojos del público francés, la versión de Arconville, por su belleza estilística y fidelidad semántica con el texto original, merece ser considerada como una más de las obras que posibilitaron la difusión de la estética de los cementerios en Francia.

\section{REFERENCIAS BIBLIOGRAFICAS}

ARConville, Thiroux Mme de, Mélanges de littérature, de morale et de physique. Tome I. Amsterdam: 1775.

BoulLLON, Duc de, Journal Encyclopedique ou Universel. Tome VIII, partie I. Bouillon: 15 novembre 1770.

DENNIS, John, Grounds of Criticism in Poetry. London: J. Darry, 1718.

DRAPER, John, The Funeral Elegy and the Rise of English Romanticism. New York: Phæton Press, 1967.

FRERON, Élie-Cathérine, L'Année Littéraire. Tome V. Paris: Delalain, 1770.

García PeINADO, Miguel A.; VelLA, Mercedes, Una modalidad singular del lirismo inglés en el siglo XVIII: "The Graveyard School". Córdoba: Servicio de Publicaciones de la Universidad, 2007.

HerveY, James, Meditations among the Tombs: In a Letter to a Lady. London: Printed for J. and J. Rivington, 1746.

HOULBROOKE, Ralph, Death, Religion and the Family in England 1480-1750. Oxford \& New York: Oxford University Press, 1998.

LÓPEZ FolgADO, Vicente, "Los cementerios de aldea, un paraje compartido en la literatura europea: de Thomas Gray a Leonor de Almeida y Unamuno", I Coloquio de Literatura Comparada, 2012.

PARISOT, Eric, "The Historicity of Reading Graveyard Poetry". En: Experiments in Genre in Eighteenth-Century Literature. Gent: Academia Press, 2011.

Pigman, George, Grief and English Renaissance Elegy. Cambridge: Cambridge University Press, 1985. 
QUERARD, Joseph-Marie, La France Littéraire ou Dictionnaire Bibliographique. Tome IV. Paris: A. Firmin Didot, 1830.

SPACKS, Patricia, "Supernatural Horror: The Atmosphere of Belief". En: The Insistence of Horror: Aspects of the Supernatural in EighteenthCentury Poetry. Cambridge \& Massachusetts: Harvard University Press, 1962.

Tourneur, Pierre Le, Méditations d'Hervey, traduites de l'anglois. Paris: Le Jay, 1771.

Van Tieghem, Paul, La Poésie de la Nuit et des Tombeaux en Europe au XVIII ${ }^{e}$ siècle. Paris: F. Rieder, 1921 (Slatkine Reprints, 1970). 